\title{
WISSENSCHAFTLICHES PERSONAL DES DEUTSCHEN ARCHÄOLOGISCHEN INSTITUTS
}

\author{
Stand am 31.12. 1968
}

\section{PRÄSIDENT}

Bittel, Kurt, Prof. Dr. phil., Berlin-Dahlem, Peter-Lenné-Str. 28-30.

\section{ZENTRALDIREKTION}

Berlin-Dahlem, Peter-Lenné-Str. 28-30. Postanschrift: Berlin 33, Postfach.

Bittel, Kurt, Prof. Dr. phil., Präsident

Grúnhagen, Wilhelm, Prof. Dr. phil., 1. Direktor,

Stellvertreter des Präsidenten

Berlin-Dahlem, Peter-Lenné-Str. 28-30.

\section{WISSENSCHAFTLICHE REFERENTEN}

Deichmann, Friedrich Wilhelm, Prof.D.Dr.phil., Wiss. Oberrat, Rom, Via Sardegna 79. - Tätig in Rom.

Buchholz, Hans-Günter, Dr. phil., Wiss. Oberrat Jessen, Hans Bernhard, Dr. phil.

Berlin-Dahlem, Peter-Lenné-Str. 28-30.

Ziegenaus, Oskar, Dr.-Ing.

\section{WISSENSCHAFTLICHE MITARBEITER}

Boehmer, Rainer Michael, Dr. phil. Freiherr von Gall, Hubertus, Dr. phil. Krien-Kummrow, Gisela, Dr. phil. Berlin-Dahlem, Peter-Lenné-Str. 28-30. Meischner, Jutta, Dr. phil. Peters, Karl, Dr. phil.

\section{MITGLIEDER DER ZENTRALDIREKTION}

Bittel, Kurt (s. Präsident).

Weickert, Carl, Prof. Dr. phil., Dr.-Ing. e. h., Berlin 45, Devrientweg 6.

Schuchhardt, Walter-Herwig, Prof. Dr. phil., Freiburg i. Br., Beethovenstr. 6.

Hampe, Roland, Prof. Dr. phil., Heidelberg, Marstallhof 4.

Schadewaldt, Wolfgang, Prof. Dr. phil., Tubingen, Nägelestr. 19. 
Goethert, Friedrich, Prof. Dr. phil., Berlin 33, Max-Eyth-Str. 29.

Schlunk, Helmut, Prof. Dr. phil., Madrid 2, Serrano, 159.

Boehringer, Erich, Prof. Dr. phil., Moosach, Haus 70.

Horn, Rudolf, Prof. Dr. phil., Göttingen, Rohnsweg 46.

Kraiker, Wilhelm, Prof. Dr. phil., Rendsburg, An der Bleiche 9.

Tschira, Arnold, Prof. Dr.-Ing., Karlsruhe, Englerstr. 7.

Drerup, Heinrich, Prof. Dr. phil., Marburg/Lahn, Biegenstr. 11.

Hagelberg, Karl-Ulrich, Ministerialdirektor im Bundesministerium des Innern, Bonn, Rheindorfer Str. 198.

Krämer, Werner, Prof. Dr. phil., Frankfurt a.M., Palmengartenstr. 10-12.

Brommer, Frank, Prof. Dr. phil., Mainz, Saarstr. 21.

Böhner, Kurt, Prof. Dr. phil., Mainz, Rōm.-Germ. Zentralmuseum.

Berve, Helmut, Prof. Dr. phil., Dr. phil. h. c., Hechendorf (Pilsensee), Rainweg 9.

Naumann, Rudolf, Prof. Dr.-Ing., Istanbul-Taksim, Sira Selvi 123.

Luschey, Heinz, Prof. Dr. phil., Teheran, Kh. Razi 93, POB. 2357.

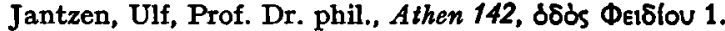

Greifenhagen, Adolf, Prof. Dr. phil., Berlin 19, Schloßstr. 1.

Kraus, Theodor, Prof. Dr. phil., Rom, Via Sardegna 79.

Himmelmann-Wildschø̂tz, Nikolaus, Prof. Dr. phil., Bad Godesberg, Körnerstr. 23.

Homann-Wedeking, Ernst, Prof. Dr. phil., Manchen 2, Meiserstr. 10.

Muller-Karpe, Hermann, Prof. Dr. phil., Frankfurt a.M., Arndtstr. 11.

Schiering, Wolfgang, Privatdozent, Dr. phil., Göttingen, Nikolausberger Weg 15.

Kaiser, Werner, Prof. Dr. phil., Kairo-Zamalek, Sharia Gezira el Wusta 22.

Otten, Heinrich, Prof. Dr. phil., Marburg/Lahn, Biegenstr. 26.

Schmidt, Hans Jargen, Prof. Dr.-Ing., Baghdad-Aiwadhiya, Sharia Ibn Duraid 135/2/1.

Otto, Eberhard, Prof. Dr. phil., Mulben ub. Eberbach.

Deichmann, Friedrich Wilhelm, Prof. D. Dr. phil., Wiss. Oberrat, Rom, Via Sardegna 79.

Hiller, Friedrich, Prof. Dr. phil., Saarbracken, Kaiserslautener Straße 83.

\section{ABTEILUNG ROM}

Rom, Via Sardegna 79.

\section{DIREKTOREN}

Kraus, Theodor, Prof. Dr. phil., 1. Direktor

Schläger, Helmut, Dr.-Ing., 2. Direktor

Rom, Via Sardegna 79

\section{WISSENSCHAFTLICHE REFERENTEN}

Blanck, Horst, Dr, phil.

Brandenburg, Hugo, Dr. phil.

Bruns, Gerda, Prof. Dr. phil., Wiss. Oberrätin

Eisner, Michael, Dr. phil.

v. Heintze, Helga, Dr. phil.

Hermann, Werner, Dr. phil.

Rakob, Friedrich, Dr. Ing.

Sichtermann, Hellmut, Dr. phil.

Völker, Hans-Herbert, Dr. phil.

Rom, Via Sardegna 79. 


\section{ABTEILUNG ATHEN}

Athen 142, dóds $\Phi_{\mathrm{E} 1 \delta l o u} 1$.

\section{DIREKTOREN}

Jantzen, Ulf, Prof. Dr. phil., 1. Direktor Willemsen, Franz, Prof. Dr. phil., 2. Direktor

Athen 142, s8ds Фetolou 1.

\section{WISSENSCHAFTLICHE REFERENTEN}

Gercke, Peter, Dr. phil. Hamdorf, Friedrich-Wilhelm, Dr. phil. Hoepfner, Wolfram, Dr.-Ing. Knigge, Ursula, Dr. phil. Mallwitz, Alfred, Dr.-Ing., Wiss. Rat Toelle, Renate, Dr. phil.

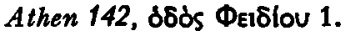

\section{RÖMISCH-GERMANISCHE KOMMISSION}

Frankfurt a.M., Palmengartenstr. 10-12.

\section{DIREKTOREN}

Krämer, Werner, Prof. Dr. phil., 1. Direktor Schönberger, Hans, Prof. Dr. phil., 2. Direktor

Frankfurt a. M., Palmengartenstr. 10-12.

\section{WISSENSCHAFTLICHE REFERENTEN}

Maier, Ferdinand, Dr. phil., Wiss. Oberrat Rochna, Otto, Dr. phil., Wiss. Bibliotheksreferent Schubert, Franz, Dr. phil., Wiss. Rat Frankfurt a.M., Palmengartenstr. 10-12. Ament, Hermann, Dr. phil., Wiss. Referent Schubert, Eckehart, Dr. phil., Wiss. Referent

\section{WISSENSCHAFTLICHE MITARBEITER}

$\left.\begin{array}{l}\text { Jacobi, Gerhard, Dr. phil. } \\ \text { Rüsch, Alfred, Dr. phil. }\end{array}\right\} \quad$ Frankfurt a. M., Palmengartenstr. 10-12.

\section{MITGLIEDER DER KOMMISSION}

Krämer, Werner, Prof. Dr. phil. Schönberger, Hans, Prof. Dr. phil. Der Präsident.

Beck, Hans, Dr. phil., Direktor, Munster/Westf., Landesmuseum far Vor- und Frahgeschichte, Rothenburg 30 . 


\section{XXVIII}

Böhner, Kurt, Prof. Dr. phil., Mainz, Römisch-Germanisches Zentralmuseum.

Dehn, Wolfgang, Prof. Dr. phil., Marburg/Lahn, Körnerstr. 1.

Drerup, Heinrich, Prof. Dr. phil., Marburg/Lahn, Biegenstr. 11.

Haarnagel, Werner, Prof, Dr. rer. nat., Wilhelmshaven, Viktoriastr. 26-28.

Hachmann, Rolf, Prof. Dr. phil., Saarbricken, Universität.

Hieronymus, Hanns, Dr. jur., Regierungsdirektor, Bundesministerium des Innern, Kulturabteilung,

Bonn, Rheindorfer Str. 198.

Jankuhn, Herbert, Prof. Dr. phil., Göttingen, Ewaldstr. 103.

Junghans, Siegfried, Dr. phil., Stuttgart, Württembergisches Landesmuseum, Altes SchloB.

Kersten, Karl, Prof. Dr. phil., Schleswig, Schloß Gottorp, Landesmuseum für Vor- und Frühgeschichte.

Kossack, Georg, Prof. Dr. phil., Kiel, Universität.

Kraft, Konrad, Prof. Dr. phil., Frankfurt a.M., Universität.

Möbius, Hans, Prof. Dr. phil., Bad Homburg v.d.H., Am Elisabethenbrunnen 17.

Nesselhauf, Herbert, Prof. Dr. phil., Konstanz, Zur Friedrichshöhe 20 b.

von Petrikovits, Harald, Prof. Dr. phil., Bonn, Colmanstr. 16, Rhein. Landesmuseum.

Schindler, Reinhard, Direktor Dr. phil., Trier, Ostallee 44, Rheinisches Landesmuseum.

Schwarz, Klaus, Dr. phil., Landeskonservator, München 22, Ottingenstr. 12.

Werner, Joachim, Prof. Dr. phil., Manchen 22, Königinstr. 69.

\section{ABTEILUNG ISTANBUL}

Istanbul-Taksim, Sira Selvi 123.

\section{DIREKTOREN}

Naumann, Rudolf, Prof. Dr.-Ing., 1. Direktor, Istanbul-Taksim, Sira Selvi 123.

2. Direktor z. Z. unbesetzt.

\section{WISSENSCHAFTLICHE REFERENTEN}

Borchhardt, Jürgen, Dr. phil.

Feld, Otto, Dr. phil.

Hauptmann, Harald, Dr. phil.

Huff, Dietrich, Dipl.-Ing.

Istanbul-Taksim, Sira Selvi 123.

\section{MITGLIEDER DES FACHAUSSCHUSSES}

Naumann, Rudolf, Prof. Dr.-Ing.

Der Präsident.

Homann-Wedeking, Ernst, Prof. Dr. phil., Munchen 2, Meiserstr. 10.

Krauss, Friedrich, Prof. Dr.-Ing., Munchen 80, Neufahrnerstr. 12.

Moortgat, Anton, Prof. Dr. phil., Berlin 37, Argentinische Allee 1.

Otten, Heinrich, Prof. Dr. phil., Marburg/Lahn, Marbacher Weg 271/2.

Unverzagt, Wilhelm, Prof. Dr. phil., Berlin 12, Sybelstr. 38.

Vogt, Joseph, Prof. Dr. phil., Tubingen, Im Rotbad 10. 


\author{
ABTEILUNG KAIRO \\ Kairo-Zamalek, Sharia Gezira el Wusta 22.
}

\title{
DIREKTOREN
}

Kaiser, Werner, Prof. Dr. phil., 1. Direktor Stadelmann, Rainer, Dr. phil., wiss. Ang. (mit der Wahrnehmung der Geschäfte des 2. Direktors beauftragt).

Kairo-Zamalek, Sharia Gezira el Wusta 22.

\section{WISSENSCHAFTLICHE REFERENTEN}

Arnold, Dieter, Dr. phil. Grossmann, Peter, Dipl.-Ing. $\} \quad$ Kairo-Zalamek, Sharia Gezira el Wusta 22.

\section{MITGLIEDER DES FACHAUSSCHUSSES}

Kaiser, Werner, Prof. Dr. phil.

Der Prăsident.

Brunner, Hellmut, Prof. Dr. phil., Tübingen, Corrensstr.

Deichmann, Friedrich Wilhelm, Prof. D. Dr. phil., Rom, Via Sardegna 79.

Helck, Wolfgang, Prof. Dr. phil., Hamburg 64, Knokenholt 5.

Kleiner, Gerhard, Prof. Dr. phil., Oberursel/Taunus, Oberhöchststadter Str. 52.

Lüddeckens, Erich, Prof. Dr. phil.. Veitshöchheim, Schillerstr. 10.

Otto, Eberhard, Prof. Dr. phil., Milben ibber Erbach.

Werner, Joachim, Prof. Dr. phil., München 22, Königinstr. 69 IV.

Zucker, Friedrich, Prof. Dr. phil., Hamburg-Großflottbek, Papenkamp 10.

\section{ABTEILUNG MADRID}

Madrid 2, Serrano, 159.

DIREKTOREN

$\left.\begin{array}{l}\text { Schlunk, Helmut, Prof. Dr. phil., 1. Direktor } \\ \text { Schubart, Hermanfrid, Dr. phil., 2. Direktor }\end{array}\right\} \quad$ Madrid 2, Serrano, 159.

\section{WISSENSCHAFTLICHE REFERENTEN}

Hauschild, Theodor, Dr.-Ing. Gamer, Gustav, Dr. phil.

Madrid 2, Serrano, 159.

\section{MITGLIEDER DES FACHAUSSCHUSSES}

Schlunk, Helmut, Prof. Dr. phil.

Der Präsident.

Dehn, Wolfgang, Prof. Dr. phil., Marburg/Lahn, Körnerstr. 1. 
$\mathrm{XXX}$

Drerup, Heinrich, Prof. Dr. phil., Marburg/Lahn, Biegenstr. 11.

Nesselhauf, Herbert, Prof. Dr. phil., Konstanz, Zur Friedrichshōhe 20 b.

Tschira, Arnold, Prof. Dr.-Ing., Karlsruhe, Englerstr. 7.

Volbach, Fritz, Prof. Dr. phil., Rom, Passeggiata di Ripetta 19.

\section{ABTEILUNG BAGHDAD}

Baghdad-Karrada, Hurriya Square $71 \mathrm{~b} / 11$.

\section{DIREKTOREN}

Schmidt, Hans Járgen, Prof. Dr.-Ing., 1. Direktor, Baghdad-Karrada, Hurriya Square 71b/11.

2. Direktor z. Z. unbesetzt.

\section{WISSENSCHAFTLICHE REFERENTEN}

Hunger, Hermann, Dr. phil. Berndt, Marianne, Dipl.-Ing.

\section{MITGLIEDER DES FACHAUSSCHUSSES}

Schmidt, Hans Jürgen, Prof. Dr.-Ing.

Der Präsident.

Berve, Helmut, Prof. Dr. phil,, Dr. phil. h. c., Hechendorf (Pilsensee), Rainweg 9.

Heinrich, Ernst, Prof. Dr.-Ing., Berlin 20, Gaismannshofer Weg 5 a.

Milojcic, Vladimir, Prof. Dr. phil., Heidelberg, Lauerstr. 1.

Moortgat, Anton, Prof. Dr. phil., Berlin 37, Argentinische Allee 1.

Schefold, Karl, Prof. Dr. phil., Basel, Spalentorweg 52.

Freiherr von Soden, Wolfram, Prof. Dr. phil., Munster/Westf., Brucknerstr. 19.

Spitaler, Anton, Prof. Dr. phil., Munchen 22, Veterinärstr. 2 III.

\section{ABTEILUNG TEHERAN}

Teheran, Khiaban Razi 93, POB. 2357.

\section{DIREKTOREN}

Luschey, Heinz, Prof. Dr. phil., 1. Direktor

Kleiss, Wolfram, Dr.-Ing., 2. Direktor

Teheran, POB. 2357.

\section{WISSENSCHAFTLICHER REFERENT}

Gropp, Gerd, Dr. phil., Teheran, POB. 2357. 


\section{MITGLIEDER DES FACHAUSSCHUSSES}

Luschey, Heinz, Prof. Dr. phil.

Der Präsident.

Berve, Helmut, Prof. Dr. phil., Dr. phil. h. c., Hechendorf (Pilsensee), Rainweg 9.

Boehringer, Erich, Prof. Dr. phil., Moosach, Haus 70.

Eilers, Wilhelm, Prof. Dr. phil., Würzburg, Theodor-Körner-Str. 6.

Heinrich, Ernst, Prof. Dr.-Ing., Berlin 20, Gaismannshofer Weg 5a.

Hinz, Walther, Prof. Dr. phil., Gōttingen, Calsowstr. 69.

Milojcic, Vladimir, Prof. Dr. phil., Heidelberg, Lauerstr. 1.

Schefold, Karl, Prof. Dr. phil., Basel, Spalentorweg 52.

Spuler, Bertold, Prof. Dr. phil., Hamburg 13, Mittelweg 90.

\section{KOMMISSION FƯR ALTE GESCHICHTE UND EPIGRAPHIK}

München 13, Schellingstraße 10 (vom 18. 12. 1969 an Amalienstraße 73)

\section{DIREKTOR}

Mit der Wahrnehmung der Geschäfte des Direktors beauftragt:

Buchner, Edmund, Dr. phil., Wiss. Oberrat, Munchen 13, SchellingstraBe 10 (vom 18. 12.1969 an AmalienstraBe 73).

\section{WISSENSCHAFTLICHE REFERENTEN}

Kolbe, Hans-Georg, Dr. phil., Wiss. Rat, Rom, Via Sardegna 79. - Tätig in Rom.

Dunst, Günter, Dr. phil. \} Wörrle, Michael, Dr. phil. $\}$

Munchen 13, Schellingstraße 10 (vom 18. 12. 1969 an Amalienstraße 73).

\section{MITGLIEDER DER KOMMISSION}

Buchner, Edmund, Dr. phil.

Der Präsident

Hieronymus, Hanns-Eberhard, Dr. jur., Regierungsdirektor, Bundesministerium des Innern, Kulturabteilung, Bonn, Rheindorfer Straße 198.

Berve, Helmut, Prof. Dr. phil., Dr. phil. h. c., Hechendorf (Pilsensee), Rainweg 9.

Bleicken, Jochen, Prof. Dr. phil., Hofheim (Taunus), Wilhelmstraße 7.

Braunert, Horst, Prof. Dr. phil., Kiel, Bülowstraße 16.

Dörner, Friedrich Karl, Prof. Dr. phil., Munster, Dodo-Str. 4 a.

Herrmann, Peter, Prof. Dr. phil., Buchholz in der Nordheide, An der Koppel 49.

Kraft, Konrad, Prof. Dr. phil., Frankfurt a. M., Am Schwalbenschwanz 35.

Lauffer, Siegfried, Prof. Dr. phil., München 22, Emil-Riedel-Straße 4.

Lippold, Adolf, Prof. Dr. phil., Regensburg, Carl-Thiel-Straße 6.

Nesselhauf, Herbert, Prof. Dr. phil., Konstanz, Zur Friedrichshöhe $20 \mathrm{~b}$.

Vittinghoff, Friedrich, Prof. Dr. phil., Köln-Lindenthal, Institut für Altertumskunde der Universität, Weyertal 92. 

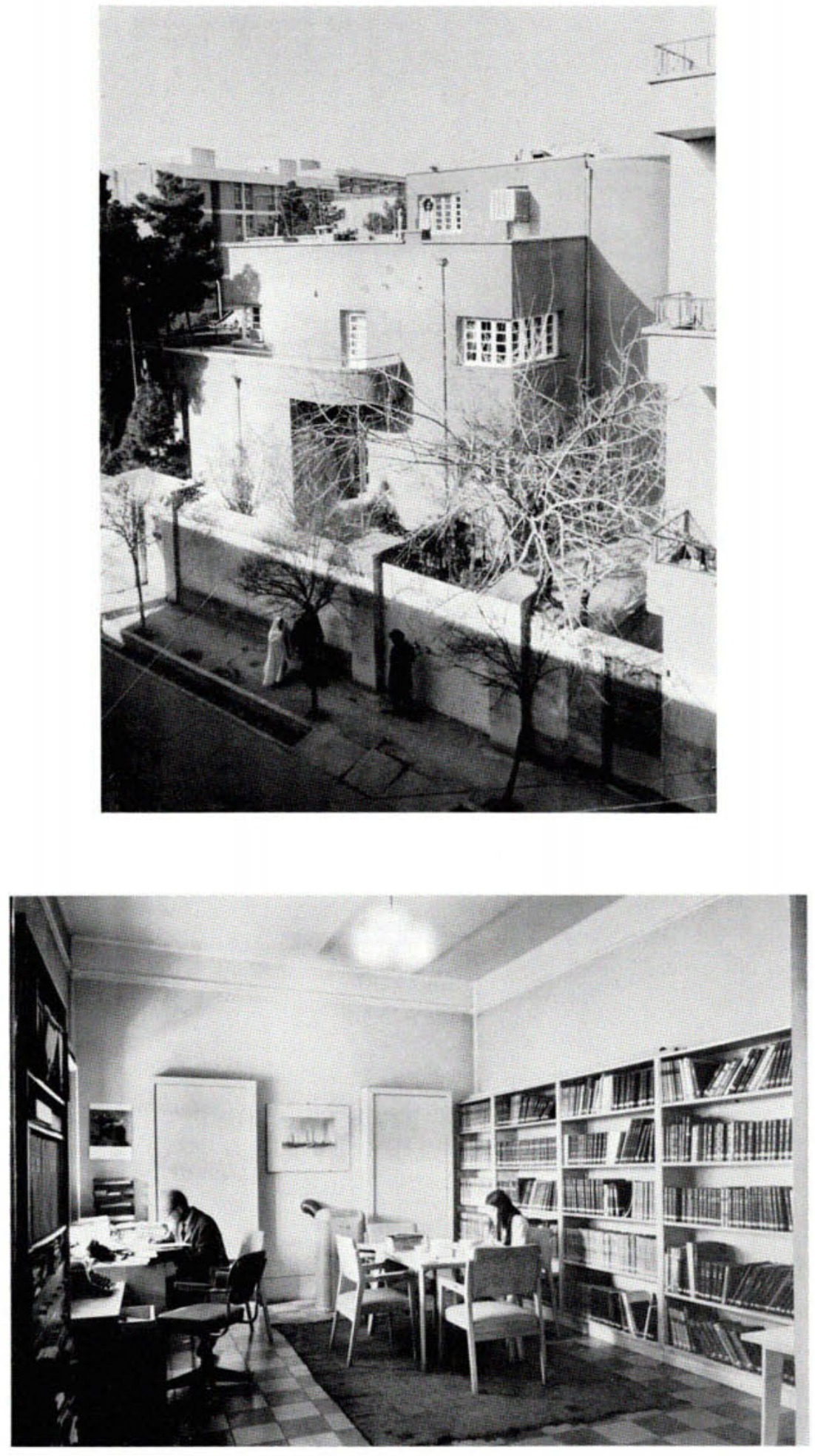

Abb. 1 und 2. Abteilung Teheran, Institutsgebäude 


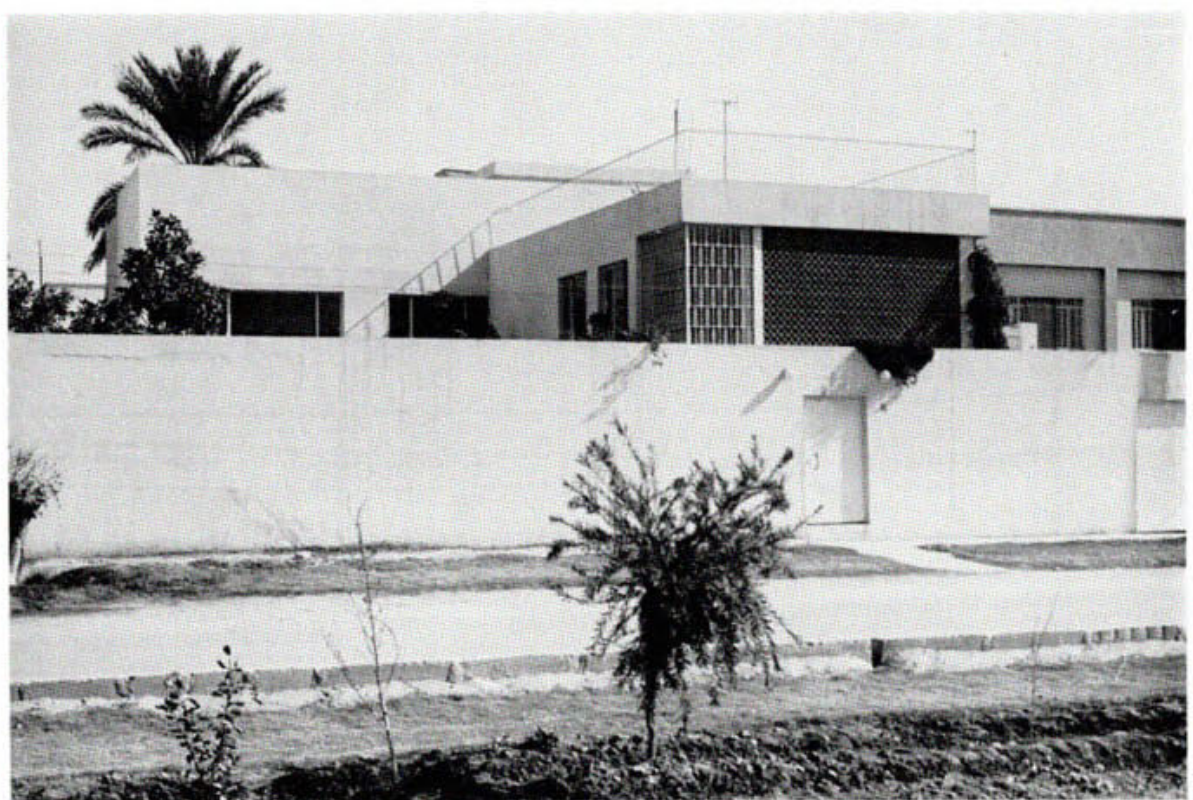

Abb. 3. Abtcilung Baghdad, neues Dienstgebäude

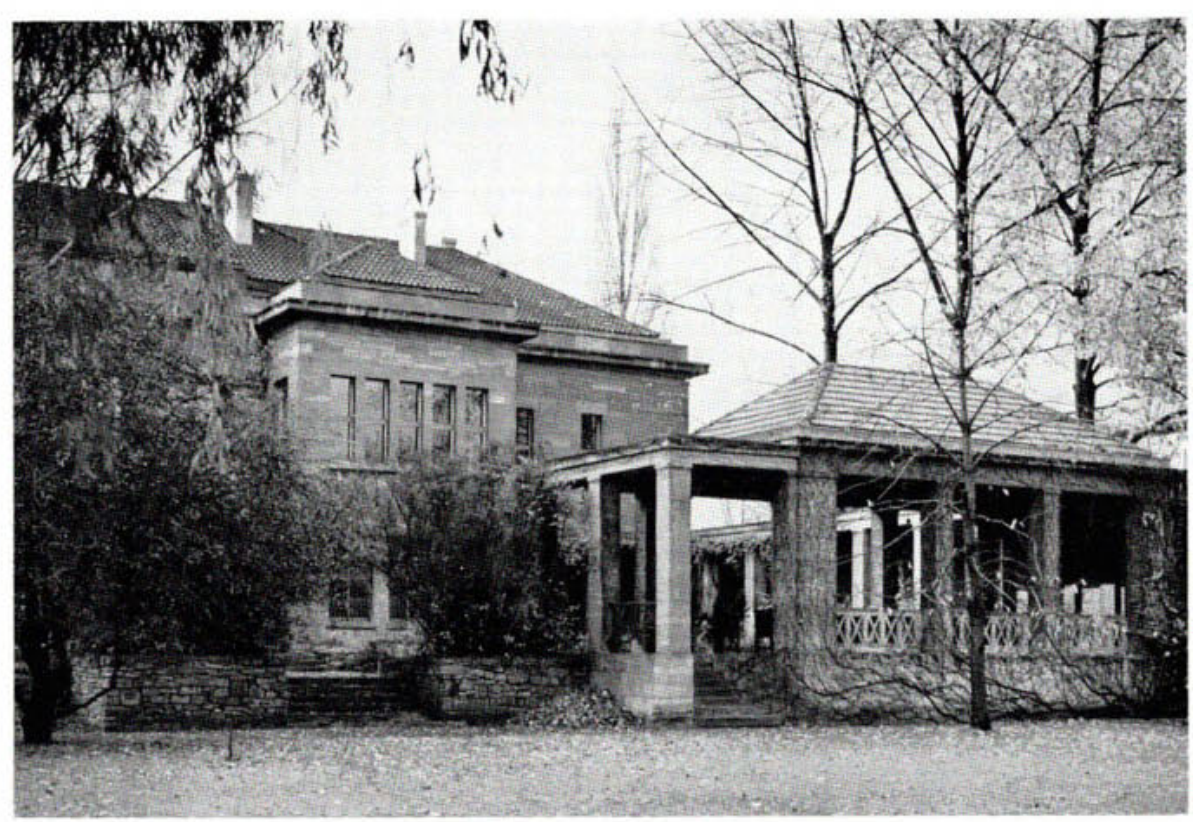

Abb. 4. Dienstgebäude der Zentraldirektion Berlin, Gartenfront 


\section{INHALT}

Jahresbericht des Deutschen Archäologischen Instituts für 1968. Mit 4 Abbildungen . . . . . . . . . . . . . . . . . . . I

Castritius, H., Die Sockelinschrift eines attischen Sarkophags in Saloniki . . . 93

Catling, H., A Mycenaean Pictorial Fragment from Palaepaphos. Mit 4 Abbildungen 24

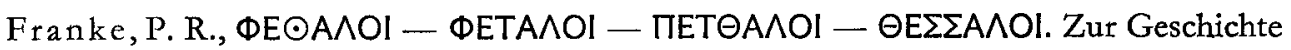
Thessaliens im 5. Jahrhundert v. Chr. Mit 26 Abbildungen . . . . . . . . . . 85

Geagan, H. A., Mythological Themes in the Plaques from Penteskouphia. Mit 19 Abbildungen .......................... 31

Karnapp, W., Die Nordtoranlage der Stadtmauer von Resafa in Syrien. Mit 18 Abbildungen . . . . . . . . . . . . . . . . . . . . 98

Kasper, S., Eine Nekropole nordwestlich von Soma. Mit einem Beitrag von W.-D. Albert und 13 Abbildungen . . . . . . . . . . . . . . . . . . 71

Schwandner, E.-L. - Ohly, D., Aegina, Aphaia-Tempel. I. Die südliche Stützmauer der Temenosterrasse. Mit 17 Abbildungen . . . . . . . . . . . . . 48

Sinos, S., Eine Untersuchung der sogenannten 'Palastanlage' von Vasiliki. Mit 36 Abbildungen . . . . . . . . . . . . . . . . . . . . . 1

Archäologische Dissertationen . . . . . . . . . . . . . . . 124 